\title{
RM21
}

\section{Using Mixtures and Skewed Distributions in Reservoir Characterization}

\section{J. Eidsvik* (NTNU)}

\section{SUMMARY}

We present two methods for dynamic reservoir characterization. Both methods are extensions of the ensemble Kalman filter (EnKF). One approach uses Gaussian mixtures and borrows ideas from the particle filter. The other approach fits a skewed Gaussian distribution from predicted ensembles. Our focus is on reliable prediction and uncertainty quantification of reservoir properties from multiple geophysical data. Elements related to value of information analysis will be discussed as well. 


\section{Introduction}

We present two methods for dynamic reservoir characterization. Both methods are extensions of the ensemble Kalman filter (EnKF). One approach uses Gaussian mixtures and borrows ideas from the particle filter. The other approach fits a skewed Gaussian distribution from predicted ensembles. Our focus is on reliable prediction and uncertainty quantification of reservoir properties from multiple geophysical data. Elements related to value of information analysis will be discussed as well.

\section{Method}

The EnKF is based on fitting a Gaussian to the predicted reservoir samples and using standard Gaussian updating with respect to data. Gaussian mixtures and skew distributions are alternatives.

The Gaussian mixture (with equal weights) is defined by

$$
p(\mathbf{x})=\frac{1}{B} \sum_{b=1}^{B} \phi_{n}\left(\mathbf{x} ; \mu^{b}, \Sigma^{b}\right)
$$

for Gaussian n-dimensional density $\phi_{n}$. When the means $\boldsymbol{\mu}^{b}$ and covariances $\Sigma^{b}$ are the same for all ensemble members, we get one Gaussian distribution. The skew normal distribution is defined by

$$
p(\mathbf{x})=2 \phi_{n}(\mathbf{x} ; \mu, \Sigma) \Phi\left(\lambda^{t} \Sigma^{-\frac{1}{2}}(\mathbf{x}-\mu)\right)
$$

for Gaussian n-dimensional density $\phi_{n}$ and univariate cumulate function $\Phi$. When the skewness vector $\lambda$ is 0 , we get the Gaussian distribution. With repeated measurements over time, we fit the mixture and skew predictive distributions from ensemble members $\mathbf{x}^{1}, . ., \mathbf{x}^{B}$. When the likelihood of the data is linear and Gaussian, both cases has closed form solutions, like in the Kalman filter.

\section{Example}

We use the reservoir simulator developed by Sintef (www.sintef.no/Projectweb/MRST/) to study the performance of the methods. The setting is a $2 \mathrm{D}$ reservoir, which initially contains a large amount of oil. Water is injected in the South-West and oil is produced in the North-East. See Figure 1 (top). We simulate one reference case of saturation, porosity and permeability, and generate seismic amplitude data at four time points using rock physics relations. Based on these simulated data we attempt to re-produce the saturation variable. In this analysis we compare the EnKF with the Gaussian mixture filter (Figure 1, bottom). The same is done for the skew filter.

\section{Conclusions}

Results show that the Gaussian mixture filter has a smaller mean square error than the EnKF. Moreover, we get a more reliable uncertainty description with the Gaussian mixture filter. The EnKF tends to undercover the variability in reservoir properties. The same results are seen for the skew filter. Value of information analysis can be used to guide the spatial and time-lapse seismic data processing for useful predictions and decision-making under uncertainty.

\section{References}

Rezaie, J. and Eidsvik, J. [2012] Shrinked (1-a) ensemble Kalman filter and a Gaussian mixture filter. Computational Geosciences, 16, 837-852.

Rezaie, J. and Eidsvik, J. [2014] Kalman filter variants in the closed skew normal setting. Computational Statistics and Data Analysis, 75, 1-14. 


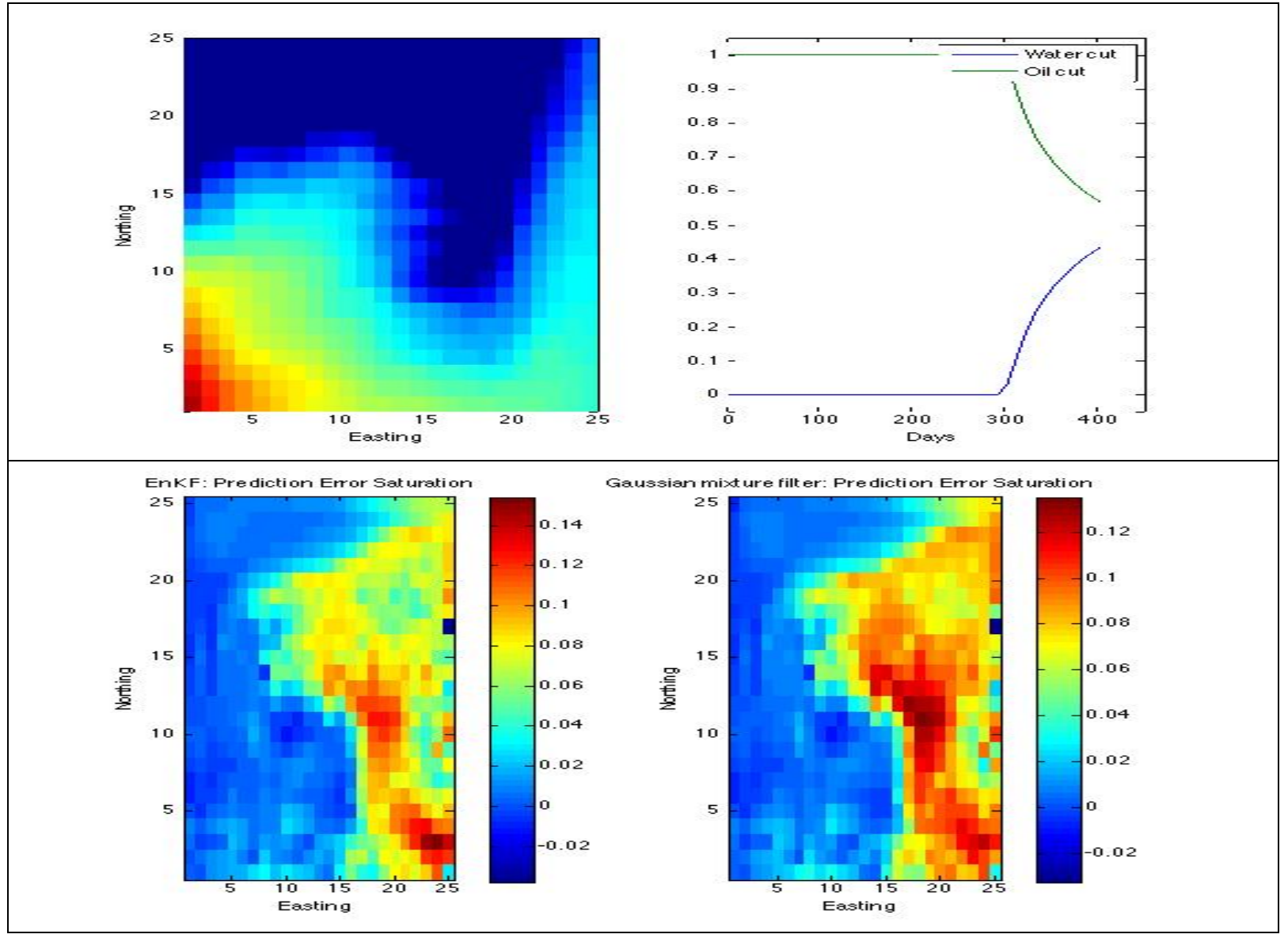

Figure 1 The reservoir simulator (top) models the flow of water from the injection well (lower left) to the production well (upper right). Both the EnKF (lower left) and the Gaussian mixture filter (lower right) have some bias in the prediction. The overall error is larger for the EnKF. 